\title{
Fatty Liver Haemorrhagic Syndrome (FLHS) in Broilers
}

\author{
Y. Ravi Kumar ${ }^{1 *}$, K. Sandhayarani ${ }^{1}$, G. Ramesh ${ }^{1}$, B. Mahesh ${ }^{1}$, G.K. Sawale ${ }^{2}$ and M. Lakshman ${ }^{1}$ \\ ${ }^{1}$ Department of Veterinary Pathology, C.V.Sc, P.V.N.R.T.V.U, Hyderabad, Telangana, INDIA \\ ${ }^{2}$ Department of Veterinary Pathology, Mumbai Veterinary College, MAFSU, Mumbai, Maharashtra, INDIA \\ *Corresponding author: RK Yadala; E-mail: ravikumaryadala@gmail.com
}

Received: 07 Sept., 2019

Revised: 17 Oct., 2019

Accepted: 27 Oct., 2019

\begin{abstract}
Fatty liver haemorrhagic syndrome (FLHS) in poultry is a metabolic disease. An outbreak of fatty liver haemorrhagic syndrome (FLHS) was detected by post mortem examination of broilers in a commercial farm. There was severe loss of production and sudden deaths with moderate mortality. Post mortem of the dead birds were performed and histopathological examination was done as per standard procedure. At necropsy, dead birds had pale combs and wattles with significant liver lesions. The liver was enlarged, friable and greasy, yellowish brown with firm fatty layer deposits and haematoma was noticed in abdominal cavity. Fat vacuoles were seen in liver sections which was also confirmed by special stain. Multiple factors like high dietary energy and stress of production may have precipitated the FLHS in broilers.
\end{abstract}

Keywords: FLHS, broilers, H\&E, Oil Red O stain

Fatty liver hemorrhagic syndrome (FLHS) is the most common non infectious cause of mortality in chickens in Northern California (Mete et al., 2013). FLHS presents clinically as sudden death in nutritionally over conditioned chicken. Pathologic examination reveals abundant coelomic fat reserves and an enlarged, pale, tan to yellow, friable liver with sub capsular and parenchymal hematomas and hemorrhages. There is often a capsular rupture with associated peracute intracoelomic suffusive hemorrhage (hemoabdomen), with a large blood clot overlaying the liver capsule and the carcass is noticeably pale due to hypovolemic anemia. Histologically, there is often hepatic lipidosis (HL) with hemorrhage and often disruption of the reticulin framework of the liver, described as reticulolysis.

The cause of FLHS has yet to be fully elucidated but is likely a combination of nutritional, genetic, environmental, and hormonal factors. It was noted early in the characterization of the syndrome that chicken that died due to FLHS were in good to obese body condition, suggesting that diet may play a role in the creation of the HL. One plausible hypothesis for the pathogenesis of FLHS is that the vacuolar swelling of hepatocytes disrupts the reticulin structure of the hepatic plates due to mechanical stress, leading to hemorrhages from the sinusoids. In addition to the mechanical stress to the liver architecture, some have suggested that the excessive lipid metabolism taking place in overfed laying hens may facilitate oxygen radical-mediated destruction of the reticulin fibers. Feeding a diet rich in antioxidants reduces the incidence of FLHS. Genetics may play an integral role in susceptibility of FLHS; in fact, others have extensively studied a strain genetically predisposed to FLHS, although a specific mechanism has not been elucidated (Yeh et al., 2009). The pathogenesis of the haemorrhage associated with this syndrome is yet to be identified. However, the haemorrhage may result from the rupture of hepatic reticulin and capsule due to the excessive liver swelling caused by accumulation of fat.

FLHS is characterised by excessive fat in the liver and haemorrhage from a ruptured liver. The syndrome occurs

How to cite this article: Kumar, Y.R., Sandhayarani1, K., Ramesh, G., Mahesh, B., Sawale, G.K. and Lakshman, M. (2019). Fatty liver haemorrhagic syndrome (FLHS) in broilers. J. Anim. Res., 9(6): 879-882. 
in caged laying hens, primarily in birds that are in positive energy balance, however other factors have also been implicated as potential contributory elements to the occurrence of FLHS. The condition is easy to recognize at necropsy with hens having excess abdominal and liver fat, haemorrhages and haematomas of various size in the liver and in many cases large blood clots in the abdominal cavity.

The present paper deals with Fatty Liver Haemorrhagic Syndrome (FLHS) in broilers. A detailed gross and microscopic pathology of FLHS was discussed.

\section{MATERIALS AND METHODS}

Fatty liver haemorrhagic syndrome (FLHS) was detected by post mortem examination of broilers in a commercial farm. It was diagnosed upon postmortem examination. The diagnosis of FLHS is based on morphological and histolopathogical analysis. The history and gross lesions were noted. Impression smears were taken for Oil Red O special staining as per standard protocol (Luna, 1968). Liver slices $\left(1 \times 1 \mathrm{~cm}^{3}\right)$ were collected and fixed in $10 \%$ neutral buffered formalin (NBF) soon after necropsy. In brief, tissues were trimmed to 3 to $5 \mu \mathrm{m}$ thickness and then dehydrated in different alcohol concentrations (70, 80, 95 and $100 \%$ ). The processed tissues were cleared in xylene and embedded in paraffin for preparation into fine blocks. Blocks were sectioned with microtome to a size of $5 \mu \mathrm{m}$; afterwards they were dewaxed and the tissue sections were stained using haematoxylin and eosin ( $\mathrm{H}$ and $\mathrm{E})$ stain. The slides were mounted with dibutylphthalate polystyrene xylene (DPX) and allowed to dry before examination under a light microscope.

\section{RESULTS AND DISCUSSION}

Gross pathology of dead birds had pale combs and wattles. The liver was highly enlarged, friable and greasy, yellowish brown with firm fatty layer deposits (Fig. 1A). A large amount of blood clots (haematoma) was also noticed in abdominal cavity. Large amount of fat deposits were also observed in abdominal cavity (Fig. 1B). Medium to large lipid vacuoles present in the impression smear of liver (Fig. 2A and 2B) and section of liver revealed small to medium vacuoles fill approximately $60-70 \%$ of hepatocytes (Fig. 3A) and distend hepatocytes diffusely throughout the tissue (Fig.3B). FLHS is currently diagnosed only after post mortem at necropsy. The gross appearance of FLHS was similar to those seen in previous investigations (Yousefi et al., 2005).

In spite of considerable research over the past few decades, the cause of the syndrome is still not completely understood. Multiple factors were incriminated in this disorder involving nutritional, environmental, genetic and hormonal factors. Overcrowding, high ambient temperature, ammonia level, cage system in commercial layer management may be predisposing birds to FLHS. As present findings, most previous investigators have
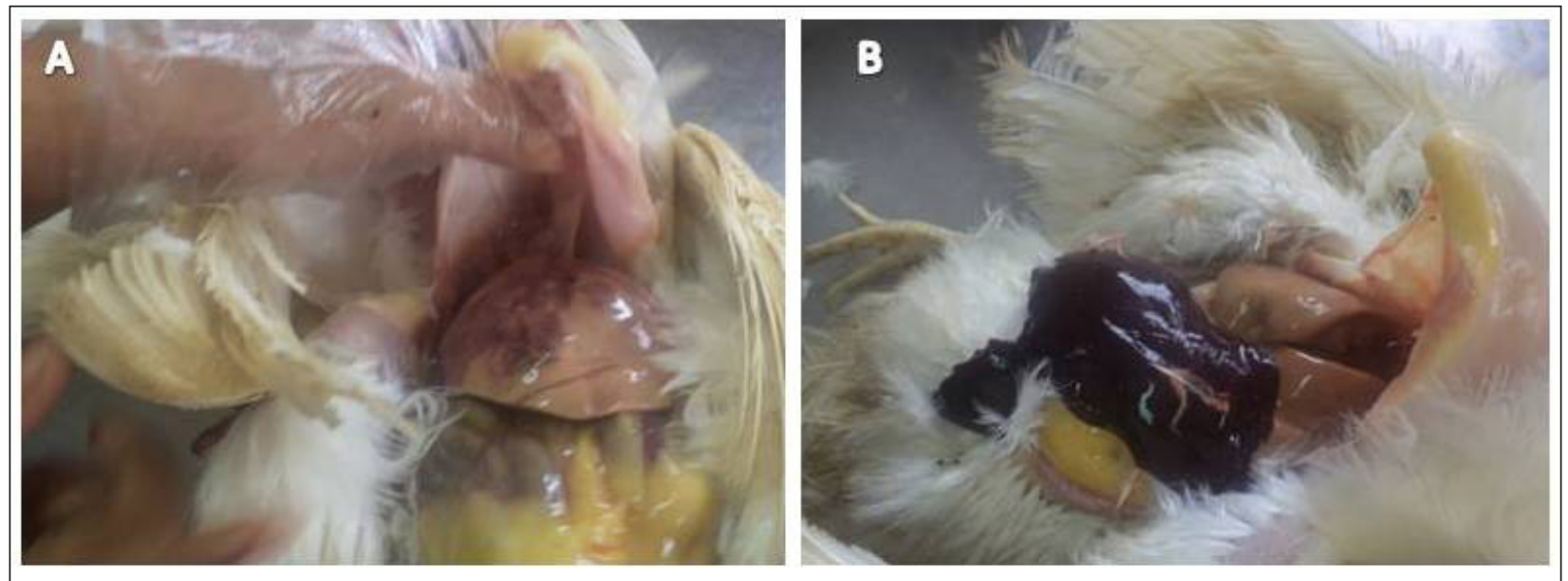

Fig. 1: Gross lesion in FLHS. (A) Enlargement of liver, pale, round boarders with fatty layer deposit; (B) Multifocal to coalescing subcapsular hepatic hemorrhages with diffuse hepatic pallor, intracoelomic blood clots and abundant intracoelomic fat 


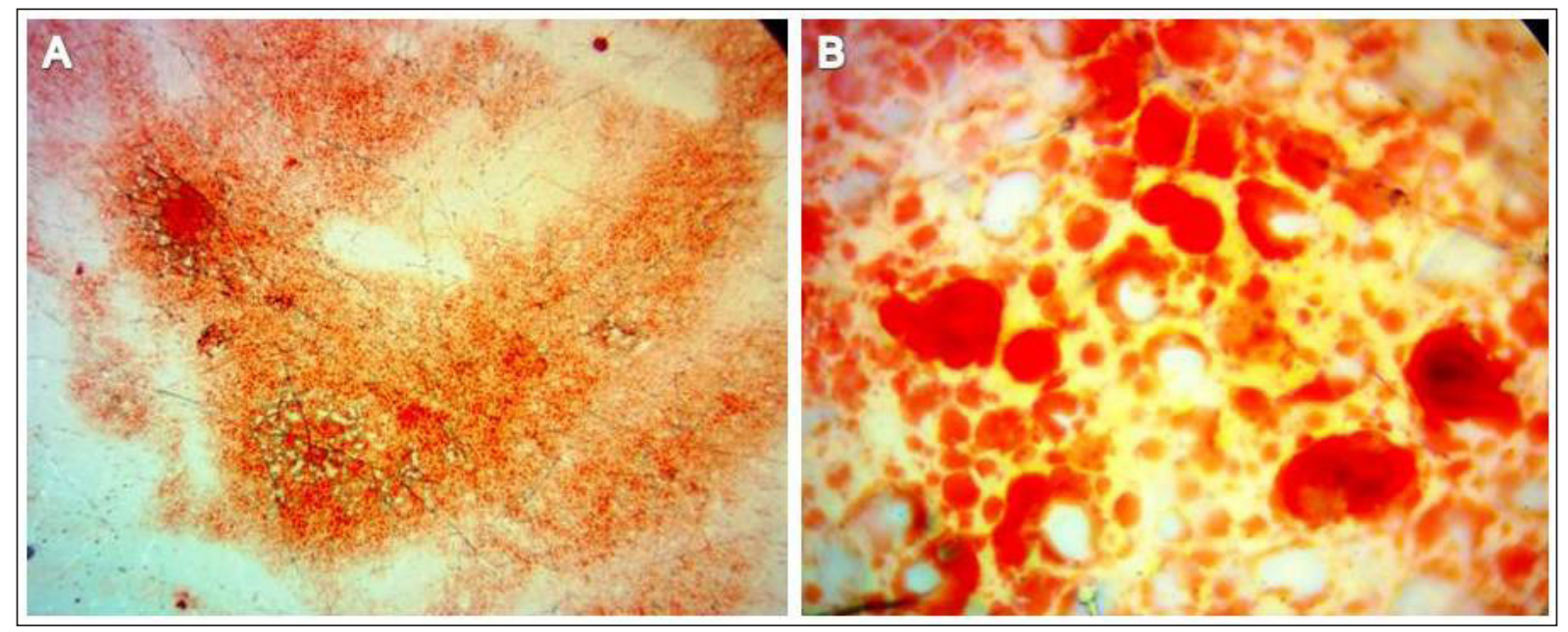

Fig. 2: Liver impression smears in FLHS. (A) Medium to large lipid vacuoles present in the impression smear of liver. Oil red O. x10; (B) Lipid vacuoles present in the impression smear of liver. Oil red O. x100.

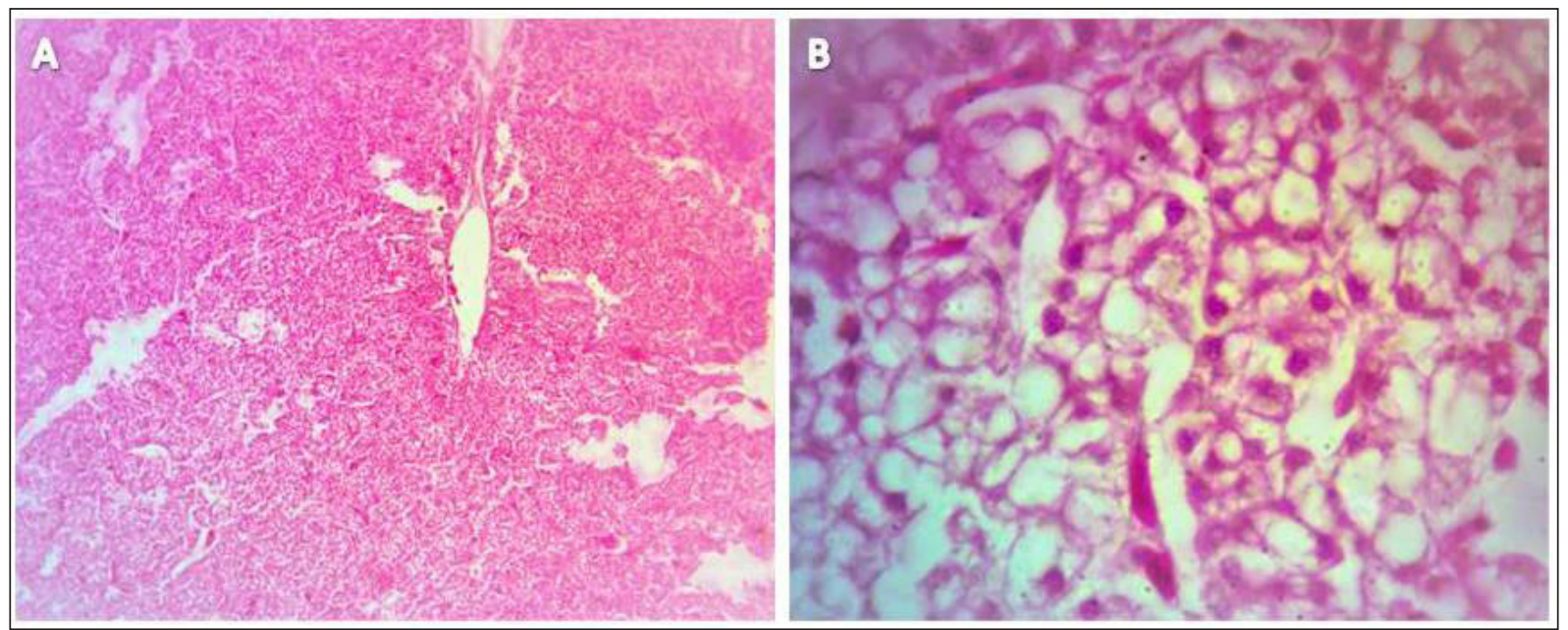

Fig. 3: Histopathological changes in the liver. (A) Photomicrograph of liver showing small to medium vacuoles fill approximately 50 $-70 \% \%$ of hepatocytes (H\&E x10); (B) Photomicrograph of liver showing small to medium vacuoles, distend hepatocytes diffusely throughout the tissue (H\&E x100).

reported that FLHS have significantly higher incidence in caged chicken than kept in other systems (Shini, 2014). However, a few studies have suggested that FLHS occurs equally in both caged and floor birds (Crespo and Shivaprasad, 2008; Trott et al., 2014). Low protein in the diet with higher metabolisable energy from fat may be predisposing to FLHS (Rozenboim et al., 2016). Higher incidence of FLHS was reported due to consumption of chelated minerals in hen than those consumed non-chelated minerals. Dietary soyabean phospholipids have been found to improve hepatic and blood lipid profile indexes relevant to FLHS (Yang et al., 2017). Dietary supplement of lipotropic agents containing choline were found helpful for remedy of FLHS (Onel et al., 2017). Supplementation of diet with Bio choline, choline chloride and lecithin extract have been found to have significantly decreased hepatic lipid concentration (Khosravinia et al., 2015). Past researchers also found increased mortality due to FLHS at higher ambient temperature but temperature factor may be of secondary importance. Exogenous estrogen in growing 
male chicks also have been found to induced hepatic fat accumulation, which might be partially due to increased lipogenic enzyme gene expression (Choi et al., 2012). Therefore, multiple factors like high dietary energy, stress and high ambient temperature may have precipitated the FLHS in broilers and need to be alleviated for effective control.

\section{CONCLUSION}

The results of study will help in better understanding the FLHS grossly and microscopically and emphasize the multiple etiological factors for effective control to prevent economical loss in broiler industry.

\section{ACKNOWLEDGMENTS}

Authors were highly grateful to PVNRTVU, Rajendranagar, Hyderabad for providing facilities to carry out present case study.

\section{REFERENCES}

Choi, Y.I., Ahn, H.J., Lee, B.K., Oh, S.T., An, B.K. and Kang C. W. 2012. Nutritional and hormonal induction of fatty liver syndrome and effects of dietary lipotropic factors in egg-type male chicks. Asian-Australasian J. Anim. Sci., 25(8): 1145 -1152 .

Crespo, R. and Shivaprasad, H.L. 2008. Developmental, metabolic, and other noninfectious disorders, In: Diseases of Poultry, 12 edition. Chap.30, Blackwell Publishing Limited, United Kingdom, pp. 1173-74.

Khosravinia, H., Chethen, P.S., Umakantha, B. and Nourmohammadi, R. 2015. Effects of lipotropic products on productive performance, liver lipid and enzymes activity in broiler chickens. Poult. Sci. J., 3(2): 113-120.
Luna, G.L.H.T. 1968. Manual of Histological and Special Staining Techniques, $2^{\text {nd }}$ Edition: 1-5. 9-34.

Mete, A., Giannitti, F. and Barr, B. 2013. Causes of mortality in backyard chickens in Northern California: 2007-2011. Avian Dis., 57: 311-315.

Onel S.E., Sunger S. and Baylan M. 2017. Effect of supplementary choline on quail meat and fatty liver. Rev. Bras. Zootec., 46(8): 645-51.

Rozenboim, I., Mahato, J., Cohen N.A. and Tirosh O. 2016. Low protein and high-energy diet: a possible natural cause of fatty liver hemorrhagic syndrome in caged White Leghorn laying hens. Poult. Sci., 95: 612-621.

Shini, A. 2014. Fatty Liver Haemorrhagic Syndrome in Laying Hens: Field and Experimental Investigations. PhD Thesis, School of Agriculture and Food Sciences, The University of Queensland, Australia. doi:10.14264/uql. 2015.74.

Trott, K.A., Giannitti, F., Rimoldi, G., Hill, A., Woods, L., Barr, B., Anderson, M. and Mete, A. 2014. Fatty liver hemorrhagic syndrome in the backyard chicken: A retrospective histopathologic case series. Vet. Pathol., 51(4): 787-795.

Yang, F., Ruan, J., Wang, T., Luo, J., Cao, H., Song, Y. Huang, J. and $\mathrm{Hu}, \mathrm{G}$. 2017. Improving effect of dietary soybean phospholipids supplement on hepatic and serum indexes relevant to fatty liver hemorrhagic syndrome in laying hens. Anim. Sci. J., 88(11): 1860-69.

Yeh, E., Wood, R.D. and Leeson, S. 2009. Effect of dietary omega- 3 and omega- 6 fatty acids on clotting activities of Factor V, VII and X in fatty liver haemorrhagic syndromesusceptible laying hens. Br. Poult. Sci., 50: 382-392.

Yousefi, M., Shivazad, M. and Sohrabi-Haghdoost, I. 2005. Effect of dietary factors on induction of fatty liver -hemorrhagic syndrome and its diagnostic methods with use of serum and liver parameters in laying hens. Int. J. Poult. Sci., 4(8): 468-72. 